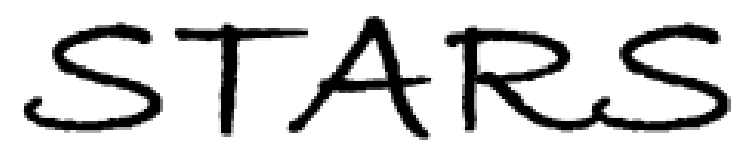

University of Central Florida

STARS

$1-1-2009$

\title{
Switchless hybrid analog-digital variable optical delay line for radio frequency signal processing
}

Nabeel A. Riza

University of Central Florida

Find similar works at: https://stars.library.ucf.edu/facultybib2000 University of Central Florida Libraries http://library.ucf.edu

This Article is brought to you for free and open access by the Faculty Bibliography at STARS. It has been accepted for inclusion in Faculty Bibliography 2000 s by an authorized administrator of STARS. For more information, please contact STARS@ucf.edu.

\section{Recommended Citation}

Riza, Nabeel A., "Switchless hybrid analog-digital variable optical delay line for radio frequency signal processing" (2009). Faculty Bibliography 2000s. 2057.

https://stars.library.ucf.edu/facultybib2000/2057

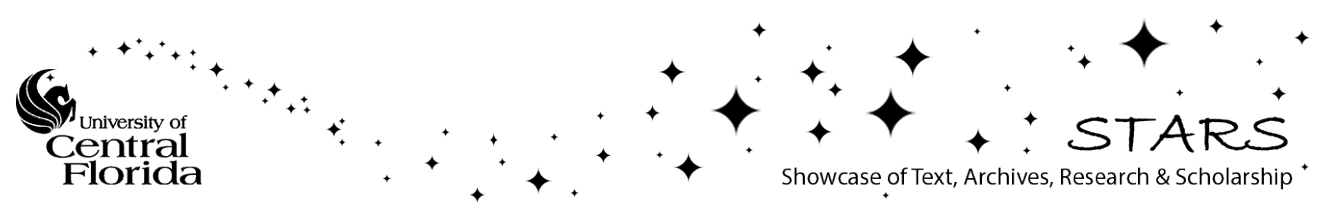




\section{Switchless hybrid analog-digital variable optical delay line for radio frequency signal processing}

\author{
Nabeel A. Riza, FELLOW SPIE \\ University of Central Florida \\ CREOL, The College of Optics and Photonics \\ Photonic Information Processing Systems \\ Laboratory 4000 Central Florida Blvd. \\ Orlando, Florida 32816-2700 \\ E-mail: riza@creol.ucf.edu
}

\begin{abstract}
The author proposes the design of a novel switchless variable optical delay line (VFODL) for radio frequency (rf) signals that combines an analog mode VFODL with a discrete-state VFODL. This hybrid VFODL proposes the use of a laser with smart, fast (e.g., 1 ns) wavelength tuning in combination with flat-top passband design spatially and temporally dispersive optical elements to simultaneously provide both long and short time delays, thus finely covering a wide nanoseconds delay range. An example design indicates a 9.6-ns time delay range with 6 -ps increments, giving 1600 independent delays using a no-movingparts signal-processing structure. VFODL applications include if antenna array processing, radar testing, and precision electrical timing systems. () 2009 Society of Photo-Optical Instrumentation Engineers. [DOI: 10.1117/1.3101455]
\end{abstract}

Subject terms: delay lines; rf photonics; phased array antenna controls; fiber optics.

Paper 090002L received Jan. 9, 2009; accepted for publication Feb. 6, 2009; published online Mar. 23, 2009.

\section{Introduction}

A variable optical delay line (VFODL) is a highly soughtafter component for radio frequency (rf) signal processing in high-resolution long-range radar and wideband communication systems. The ideal VFODL for these applications is able to efficiently and continuously generate time delays with high (e.g., picoseconds) temporal resolution over a long (e.g., nanoseconds) delay range. Over the years, efforts have been made to realize these VFODLs for $\mathrm{rf}$ applications. ${ }^{1}$ One way to efficiently generate variable time delays over a long delay range uses an $N$-bit switched binary architecture that employs $2 \times 2$ digital switches to select given binary paths connected in a serial architecture. Free-space, solid-optic, and fiber-based delay paths have been deployed in architectures using switching technologies like liquid crystals, ${ }^{2}$ lithium niobate integrated optics, ${ }^{3}$ and microelectromechanical systems (MEMS).

Because these VFODLs are digitally switched, their time delay resolution is quantized to a discrete value, and there is a trade-off between resolution and number of binary switched stages. In effect, getting higher resolution across larger time delay ranges means adding more cascading, leading to higher losses and greater VFODL complexity. Hence a dilemma exists in achieving a VFODL with both high resolution and long time delay range without drastic accumulations in loss and crosstalk.

An attractive technology for generating time delays involves the use of wavelength tuning and fiber Bragg gratings (FBGs). ${ }^{4,5}$ Initially discrete FBGs positioned along specified fiber paths were used to produce discrete time delays based on the chosen wavelength. The concept was

0091-3286/2009/\$25.00 @ 2009 SPIE also extended to a chirped FBG to generate near-continuous time delay, but only over a short delay range, due to the fabrication size limitations of FBGs and the laser's tuning range. For more delay settings within an efficient structure, multiwavelength fiber time delay processing was proposed, using discrete FBG delay segments within a serial switched structure. ${ }^{6}$ In a similar vein, proposed is the use dispersive fibers and wavelength tuning to get delays, although a long time delay range (many nanoseconds) would need many kilometers of dispersive fiber, which would add to the VFODL's weight and temperature sensitivity. ${ }^{7,8}$ In addition, wavelength tuning in combination with wavelength division multiplexer (WDM) devices has been proposed to realize VFODLs. ${ }^{9-11}$ So far, such efforts, to the best of our knowledge, have not realized an rf-application VFODL that can deliver near-continuous time delays over a large range at fast (nanosecond) speeds, in addition to providing low (e.g., $<-35 \mathrm{~dB}$ ) optical crosstalk levels, graceful accumulation of loss, and minimal weight and size. This paper describes an attempt to realize such a VFODL.

\section{Design of a Switchless Hybrid Analog-Digital Variable Optical Delay Line}

Figure 1 shows the proposed VFODL ${ }^{12}$ with rf input and output ports and one delay control port. The proposed structure is a cascade of an efficient digitally switched VFODL in combination with an analog-controlled VFODL. This hybrid combination solves the earlier resolution-range dilemma, because the digital delay is excellent for providing the long time-delay range while the analog delay is excellent in providing the near-continuous high-resolution delay between the discretized delays. An earlier version of this hybrid VFODL scheme used slow (millisecond) optomechanical fiber optical switches to form the digitally 


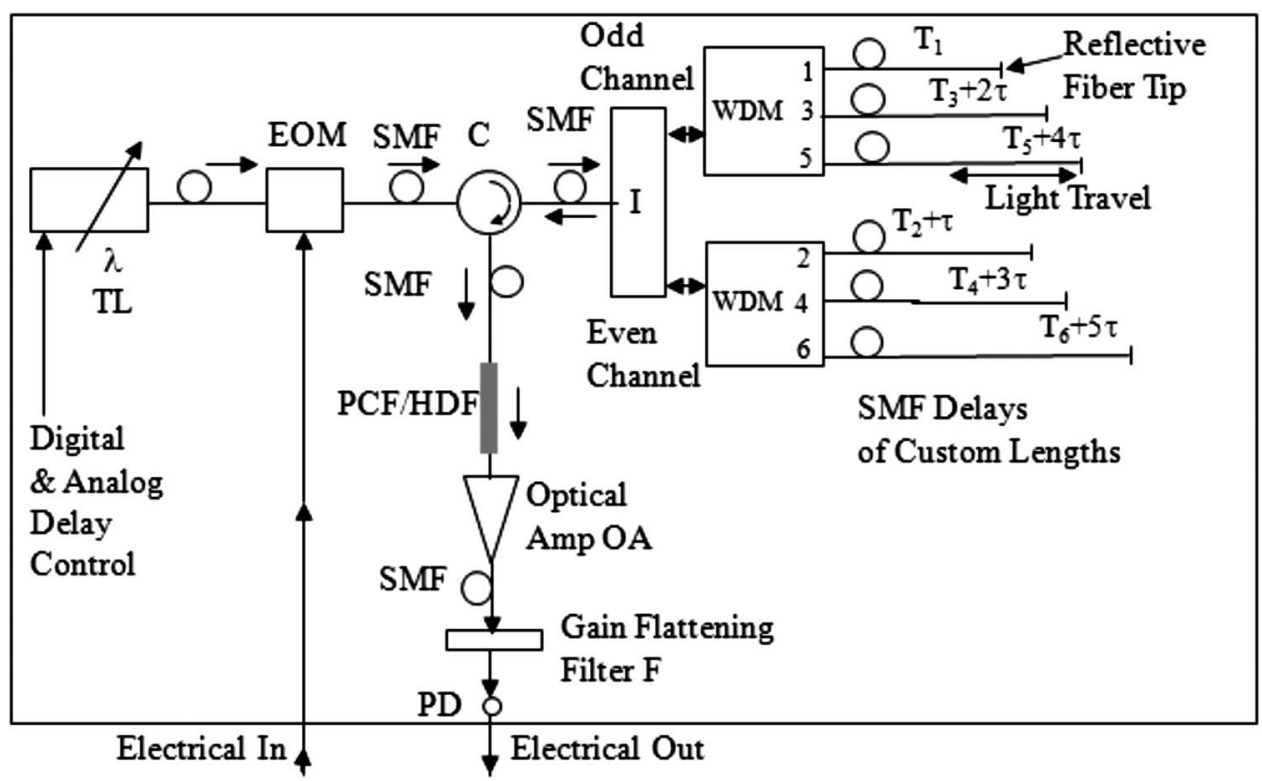

Fig. 1 The proposed switchless VFODL realized as a serial-parallel wavelength-coded digital-analog delay line, shown here using $N=6$ discrete channels. TL: tunable laser; EOM: rf-to-optical modulator; PD: photodiode detector; PCF: photonic crystal fiber; HDF: high-dispersion fiber; C: optical circulator; WDM: flat-top wavelength division multiplexer device; I: interleaver.

switched delay line part of the VFODL, and a chirped FBG to form the analog delay line, leading to a 22-bit delay performance. ${ }^{13}$ The proposed VFODL is an innovation within the hybrid analog-digital theme, which importantly does not require traditional fiber optical switches, thus leading to a switchless VFODL with graceful loss accumulation and low crosstalk that can realize superfast (nanosecond) domain delay selection via smart laser tuning. Specifically, light from a fast-tuning tunable laser (TL) chip is intensitymodulated at rf using an integrated electro-optic modulator (EOM) chip. The light then passes via a single-mode fiber (SMF) and a three-port optical circulator (C) to enter an interleaver (I) device that splits the light into $N / 2$ odd and
$N / 2$ even wavelength channels, with each interleaver output port connected to an N/2-channel WDM device.

The WDM device can be made of various elements, including FBGs, thin-film filters, free-space-coupled bulk gratings, photonic crystal prisms, and arrayed waveguide gratings. In our case, the WDM devices are flat-top passband devices (Fig. 2) with each device coupling to $N / 2$ custom-length fiber delay lines. ${ }^{14}$ The use of odd and even channels and two WDMs improves interchannel crosstalk performance, because effectively the interchannel wavelength separation between fiber channels in each WDM is doubled compared to a single WDM with an $N$-channel

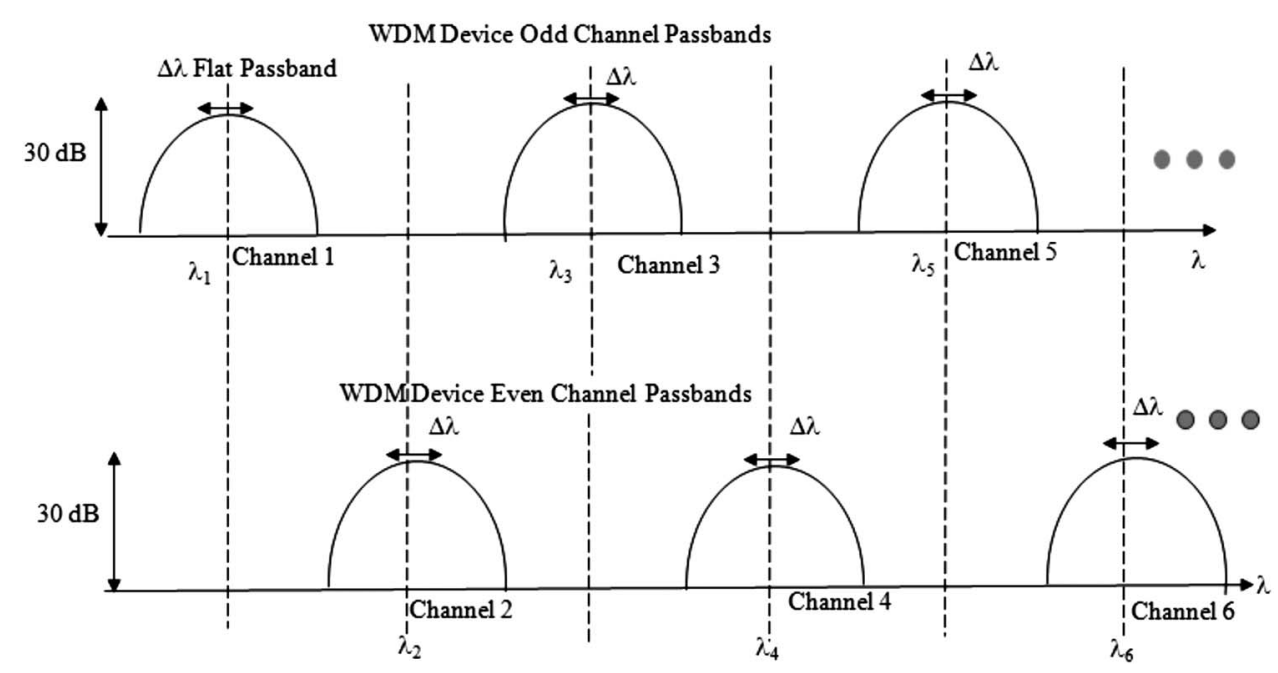

Fig. 2 Required flat-top passband of the two WDM devices used in the Fig. 1 VFODL design. Example shows three channels per WDM device. 
count over the same full fiber channel access optical bandwidth. The custom-length SMFs parallel array is terminated with fiber mirrors (FMs), which act to reflect the light back through the WDM devices to the single high-dispersion fiber (HDF) channel analog delay line in the VFODL. All fibers used in the digital part of the delay line design are regular low-dispersion SMFs forming short compact wavelength-independent fiber segments. The WDMs are designed to have flat-top $\Delta \lambda$ passbands, so one can tune the laser by $\pm 0.5 \Delta \lambda$ around any one of the $N$ central wavelengths $\lambda_{1}, \lambda_{2}, \lambda_{3}, \ldots, \lambda_{N}$, and still have full optical coupling into the wavelength-selected SMFs connected to the chosen WDM. The central wavelength position number (also corresponding to the WDM channel number) is given by $n=1,2,3, \ldots, N$. The odd-wavelength WDM device, via its custom-cut SMFs, gives time delays of $T_{1}, T_{3}+2 \tau, T_{5}$ $+4 \tau, \ldots, T_{N-1}+(N-1) \tau$ for wavelengths set to $\lambda_{1} \pm 0.5 \Delta \lambda$, $\lambda_{3} \pm 0.5 \Delta \lambda, \lambda_{5} \pm 0.5 \Delta \lambda, \ldots, \lambda_{N-1} \pm 0.5 \Delta \lambda$, respectively. Similarly, the even-wavelength WDM device via its custom cut SMFs gives delays of $T_{2}+\tau, T_{4}+3 \tau, T_{6}+5 \tau, \ldots, T_{N}$ $+(N-1) \tau$ for wavelengths set to $\lambda_{2} \pm 0.5 \Delta \lambda, \lambda_{4} \pm 0.5 \Delta \lambda$, $\lambda_{6} \pm 0.5 \Delta \lambda, \ldots, \lambda_{N} \pm 0.5 \Delta \lambda$, respectively. Here the bias delay is $T_{n}=T_{0}+(n-1) T_{b}$, where $T_{0}$ is a chosen fixed fiber length that enables time delay referencing and simple fiber interconnection assembly. The delays $(n-1) T_{b}$ are the WDM channel-specific bias delays provided by the precut bias SMF lengths that remove the relative delay referenced to wavelength $\lambda_{1}$ (or WDM channel $n=1$ ), because all light after delay processing through the WDMs must pass through the HDF analog delay line, which provides a wavelength-dependent delay of $D L \lambda$, where, the HDF has a length $L$ and dispersion $D$.

On selecting a central wavelength number $n$, light flows to a given discrete SMF delay connected to either the odd or the even WDM device, hence picking up the long discretized time delay. This delayed light, after retroreflection in its specific SMF segment, returns via the interleaver and circulator to enter the HDF analog delay line. As shown in Fig. $1, D$ is a negative dispersion, say $-600 \mathrm{ps} /(\mathrm{nm} \mathrm{km})$, such as might be obtained from a specialty photonic crystal fiber (PCF), e.g., from A/S Fiber (Blaze Photonics), Denmark. To fine-tune the discrete long delay within a delay increment $\tau$, the laser is tuned by $\Delta \lambda$ about the given central wavelength. Because, as mentioned earlier, this analog tuning effect should add the same time delay tuning regardless of the central wavelength selected, any relative central wavelength change delay due to the HDF is canceled by the specific bias delays $T_{n}$ with $T_{b}=L D\left(\lambda_{2}-\lambda_{1}\right)$. Note that, depending on whether $D$ is negative or positive, one must place the bias delays in the appropriate order along the WDM device channels to cancel the channel-to-channel DHF dispersion-based delay skew HDF. The $n=1$ WDM port provides the analog-only 0-to- $\tau$ delay, while all the other WDM ports $(n=2,3, \ldots, N)$ provide analog + digital-controlled delays from $\tau$ to $N \tau$. This completes the delay operation of the proposed VFODL, which will give a delay dynamic range of $N \tau$, with incremental digital steps $\tau$ and with precision tuning limited by the tuning resolution of the laser.

An important point to note in the VFODL design in Fig. 1 is the use of a fixed-gain flattening optical filter $F$ in combination with an optical amplifier (OA). Because each WDM device, and for that matter the entire VFODL, has a certain imperfect spectral response that can be measured, one can place a custom-gain flattening filter $F$ in cascade with a high-gain (e.g., 20 to $30 \mathrm{~dB}$ ) OA to remove nonuniformity in the optical signal gain or loss after time processing through the VFODL. In effect, one can make a nearly lossless VFODL that produces the same level of delayed optical and rf signals regardless of the spectral wavelength selected for delay processing. This design advance provides a powerful mechanism for enabling the proposed hybrid VFODL solution.

\section{Application of the Proposed VFODL in RF Array Antenna Controls}

High-resolution long-range phased array radars operate typically in the $\mathrm{L}, \mathrm{S}$, and $\mathrm{C}$ (1 to $8 \mathrm{GHz}$ ) radar bands with aperture sizes cf several meters. In effect, one needs long (10-ns) delays with more than 8 bits of resolution for accurate wideband beam steering. To design the proposed VFODL for such a radar, one can use a PCF available today, which in a 1-km loop gives a 600-ps delay with the analog wavelength tuned over a bandwidth $\Delta \lambda=1 \mathrm{~nm}$. As a result, one can design the WDM-linked SMF delay step to be $\tau=600 \mathrm{ps}$, a highly practical short $(12 \mathrm{~cm}) \mathrm{SMF}$ length increment that forms a nearly wavelength-independent waveguide that can also be packaged for light weight and robust environmental controls. One can pick $\lambda_{n+1}-\lambda_{n}$ $=2 \mathrm{~nm}$, so the separation between channels in each WDM is $4 \mathrm{~nm}$, which is excellent for low-crosstalk $(-40 \mathrm{~dB})$ performance. For example, the typical optical loss of a Photeon, Inc. flat-top WDM eight-channel device with 1.6-nm interchannel separation is $3.3 \mathrm{~dB}$, with excellent nonadjacent-channel (3.2 nm away) optical crosstalk $(-43 \mathrm{~dB}){ }^{15}$ The 2 -nm interchannel gap corresponds to the bias delay increment $T_{b}$ of a 24-cm SMF length. If $N=16$, the proposed VFODL design gives a 9.6-ns time delay dynamic range with 16 discrete steps, each with a 600-ps resolution, using two typical eight-channel WDM devices. Given a tunable laser with a tuning resolution of $0.01 \mathrm{~nm}$, one gets 100 steps within the 1-nm analog tuning range. Overall, the laser has to be tuned over $(N-1)\left(\lambda_{n+1}-\lambda_{n}\right)$ $+\Delta \lambda=31 \mathrm{~nm}$, a bandwidth possible with today's tunable lasers.

Thus, one has realized a VFODL with 1600 states and with more than 10 bits of delay control. More importantly, this switchless VFODL design can operate at extremely fast (nanosecond) speed, because it sets delays via a nomoving-parts fast-tuning laser. ${ }^{16}$ The double-pass $6.6-\mathrm{dB}$ optical loss of the WDM device plus other fiber optic components such as $\mathrm{C}$, I, and PCF/HDF, and F are compensated by the $\mathrm{OA}$ with gains from 20 to $30 \mathrm{~dB}$, thus potentially leading to an optically lossless VFODL design. Also note that there are no moving parts in the entire Fig. 1 design, promising robustness for deployment on mobile platforms such as aircraft and ships. Finally, this VFODL can be scaled to more bits using additional WDM devices, interleavers, and wider-bandwidth, higher-resolution tunable lasers.

In deployed antenna systems, temperature and mechanical stresses (including $g$ forces) play a big environmental 
role that requires constant calibration and time delay error monitoring over beamformer operational lifetimes. In this case, any extra bits beyond 7 come in very handy for critical beamformer calibration for accurate radar beam pointing. Also, as the antenna array element count increases and the beamformer needs to perform both 1-D and 2-D beam steering, the rf time delay errors in the beamformer system get worse. Hence it is all the more important to have a time delay unit (TDU) per antenna element for these wideband arrays. Thus the proposed VFODL is ideal for an rf array antenna beamformer design that uses the robust perantenna-element TDU architecture.

\section{Conclusion}

The proposed VFODL design is a switchless all-passive design that has the potential to save power, weight, and cost associated with complex optical switching hardware in beamformers. Specifically, the focus of the VFODL controls is shifted to the remote single-site laser control electronics rather than a distributed switch such as a complex optical MEMS space switch or a cascaded integrated-optic switching structure. The VFODL has promise for various electrical signal conditioning applications, and future work will describe experimental implementations.

\section{References}

1. N. A. Riza, Ed., Selected Papers on Photonic Control Systems for Phased Array Antennas, SPIE (1997).

2. L. Pastur, S. Tonda-Goldstein, D. Dolfi, J. Huignard, T. Merlet, O Maas, and J. Chazelas, "Two-dimensional optical architectures for the receive mode of phased-array antennas," Appl. Opt. 38 (14), 3105 3111 (1999).

3. R. A. Soref, "Programmable time-delay devices," Appl. Opt. 23 (21), 3736-3737 (1984)

4. R. A. Minasian, "Photonic signal processing of high-speed signals using fiber gratings," Opt. Fiber Technol. 6 (2), 91-108 (2000).

5. J. Capmany, D. Pastor, and B. Ortega, "New and flexible fiber-optic delay-line filters using chirped Bragg gratings and laser arrays," IEEE Trans. Microwave Theory Tech. 47 (7), 1321-1326 (1999).

6. N. A. Riza and N. Madamopoulos, "Phased-array antenna, maximum-compression, reversible photonic beam former with ternary designs and multiple wavelengths," Appl. Opt. 36 (5), 983-996 (1997).

7. R. Soref, "Optical dispersion technique for time-delay beam steering," Appl. Opt. 31 (35), 7395-7397 (1992).
8. R. D. Esman, M. Y. Frankel, J. L. Dexter, L. Goldberg, M. G. Parent D. Stillwell, and D. G. Cooper,"Fiber-optic prism true time delay antenna feed," IEEE Photonics Technol. Lett. 5 (11), 1347-1349 (1993).

9. S. Yegnanarayanan, P. D. Trinh, and B. Jalali, "Recirculating photonic filter: a wavelength-selective time delay for phased-array antennas and wavelength code-division multiple access," Opt. Lett. 21 (10), 740-742 (1996).

10. N. A. Riza and S. Sumriddetchkajorn, "Micromechanics-based wavelength-sensitive photonic beam control architectures and applications," Appl. Opt. 39 (6), 919-932 (2000).

11. V. Polo, B. Vidal, J. L. Corral, and J. Marti, "Novel tunable photonic microwave filter based on laser arrays and $N \times N$ AWG-based delay lines," IEEE Photonics Technol. Lett. 15 (4), 584-586 (2003).

12. N. A. Riza, "Hybrid photonic signal processing modules," U.S. Patent Pending.

13. N. A. Riza, M. A. Arain, and S. A. Khan, "Hybrid analog-digital variable fiber-optic delay line," J. Lightwave Technol. 22 (2), 619 624 (2004)

14. Y. Akahane, T. Asano, H. Takano, B. Song, Y. Takana, and S. Noda, "Two-dimensional photonic-crystal-slab channel drop filter with flattop response," Opt. Express 13 (7), 2512-2530 (2005).

15. Photeon, Inc., Bregenz, Austria, data sheet for $200-\mathrm{GHz} 8$-channel flat-top DWDM MUX/DEMUX (2008).

16. G. Alibert, F. Delorme, P. Boulet, J. Landdreau, and H. Nakajima, "Subnanosecond tunable laser using a single electroabsorption tuning superstructure grating," IEEE Photonics Technol. Lett. 9 (7), 895-897 (1997).

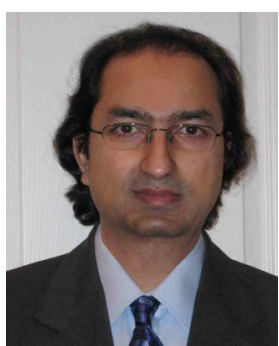

Nabeel Riza holds a doctorate from the California Institute of Technology. In 2002, he became the first Caltech Alumnus to be awarded the prestigious International Commision for Optics (ICO) Prize and coawarded 2001 Ernst Abbe Medal from the Carl Zeiss Foundation, Germany. His other awards include 2007 IEEE Fellow Award, 1998 OSA Fellow Award, 1998 International Society for Optical Engineering (SPIE) Fellow Award, 2008 Illinois Institute of Technology (IIT) Distinguished Alumni Professional Achievement Award, and 2008 Berthold Leibinger Innovation Prize Nominee DistinctionGermany. After completing his PhD in 1989, Riza joined the Genera Electric (GE) Corporate Research and Development Center, New York, where he initiated and led the GE Optically Controlled Radar Project. In 1995, he joined CREOL, The College of Optics \& Photonics at the University of Central Florida where he is full professor and head of the Photonic Information Processing Systems Laboratory. For 2007-2008, he was selected as an European Union Erasmus Scholar Visiting Professor at the Delft University of Technology, Netherlands. 